\title{
Sound Speed Measurements in solids: Absolute Accuracy of an Improved Transient Pulse Method
}

\author{
Thomas M. Proctor, Jr. \\ Institute for Basic Standards, National Bureau of Standards, Washington, D.C. 20234
}

(November 17, 1970)

\begin{abstract}
A modified transient pulse technique for measuring shear and longitudinal sound speeds in solids has been devised. The technique is described and evaluated for both precision and accuracy on a variety of solids. This evaluation has been done by experiments in which the constancy of sound speed with path length is used as the prime test for accuracy. A number of variables such as transducer frequency, transducer size, choice of different coincidence points, etc., have been examined. The transducer bond problem is examined and overcome by the use of a time delay bond. Indicated accuracy of the method is found to be better than one part in $10^{4}$. Advantageous sample geometries for accuracy testing are discussed.
\end{abstract}

Key words: Acoustical pulse; longitudinal and shear sound waves; solids sound speed accuracy; speed of sound; transient pulse-acoustical.

\section{Introduction}

For the purpose of this discussion, measurement methods for sound speed can be divided into two groups: (1) equilibrium-monochromatic sinusoidal methods (ES), and (2) nonequilibrium sharp-transient pulse methods (NET). For the ES methods, error evaluation depends upon their described character. The specification of the particular equilibrium condition is important. Many variations of the ES method are discussed in the extensive works of H. J. McSkimin and others [1-5]. ${ }^{1}$ But in the NET methods the predominant notion is that the first sound arrival proceeds by the shortest path and travels with the sound speed of the infinite medium. The nearer to the first arrival that the measurement is done, the less significant are the various end effects and unwanted boundary reflections.

Because the NET method performs poorly for materials that have frequency dispersion or high losses, its use has been limited in recent years. The monochromatic ES systems are more applicable to these cases. Despite these limitations, various NET methods have been used successfully in this laboratory [6-8]. Recently, the latter has been applied to the measurement of speed of sound in solids with surprisingly good results. Modifications of an older technique have largely overcome the remnant sound energy problem which occurs with continuous pulsing.

The method uses a pair of very short pulses, each approximating a delta function. The pulses are separated by a time interval which is precisely adjusted to correspond to the round-trip time of flight for sound through the sample. The signal pair is followed by a dead time which is sufficiently long enough for the residual sound to have died out. During this dead time, the transducer is used as a receiver in such a manner that the $n^{\text {th }}$ return

\footnotetext{
1 Figures in brackets indicate the literature references at the end of this paper.
}

of the second pulse is superimposed on the $(n+1)^{\text {th }}$ return of the first pulse. The earliest recognizable feature of these returning signals is used to make this coincidence. This is related to the most direct path for sound travel. This contrasts sharply with the ES methods. In general, the phase lag of the acoustical signal is measured by comparison to the infinitely long wave train from which the initial pulses are chopped. These ES experiments are essentially monochromatic, as the propagated pulses are made up of many wavelengths in signals of constant amplitude.

The method is discussed with special emphasis on precision and accuracy as determined empirically. The uniformity of the sound speed and the similarity between the two pulses used for coincidence were used as tests for accuracy. The effects of different variables on the sound speed have been examined and are noted. Some guidelines (shape and material) for the choice of a suitable sample for testing the accuracy of the method have been developed.

\section{Experimental Features}

This transient video pulse technique is used for sound speed measurements in short fat solid samples $(3.5 \mathrm{~cm}$ long $\times 3.8 \mathrm{~cm}$ diam) and can be used either single ended or with separate sender and receiver. The single ended way produces the best results and is used for this study. The transit time for a round trip is the principal measurement made.

With continuous pulsing at intervals comparable to the transit time of the sample, remnant sound will build up in small samples of high $Q$ and coincidences for timing the transit time will be impaired. The remnant sound problem is handled by the use of a special sequence of driving pulses. The transducer is driven by two very short 


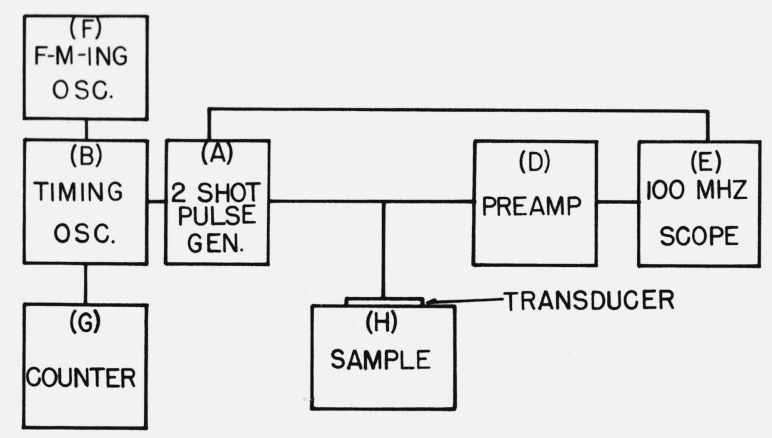

Figure 1. Experimental setup schematic.

( $<30 \mathrm{~ns}) 50-\mathrm{V}$ pulses, each approximating a delta function, which are separated by a known and adjustable time period, $\tau$. These pulses are followed by a long dead time $\left(2^{n}-1\right) \tau$ and then the full sequence is repeated. The period $\tau$ is determined by the frequency of the control oscillator (B) as measured by the frequency counter (G) (see fig. 1). This drives the two-shot apparatus (A) which produces the pulse sequence.

The dead time generated by the two-shot apparatus can be adjustable to any value $\left(2^{n}-1\right) \tau$ where $n$ is an integer ranging from 1 to 8 . Such adjustments allow for variations in sample losses by permitting the dead time to be adjusted so that, at the beginning of the next sequence, the left-over residual sound is negligible. Most of these measurements were made with $n=8$ and with a dead time of $255 \tau$. The two-shot apparatus is a parallel set of $1 \mathrm{MHz}$ binary chains having an adjustable phase difference for pulses traveling down the two chains [9] (see fig. 2). A provision which allows interchanging the firing order of the respective chains is used to cancel slight electronic phase differences between the outputs of the two chains. This function is indicated by the "A before B" switch on figure 2 .

The pulses are then amplified and fed to a blocking oscillator which drives the transducer. The transducer, acting as a highly damped resonant sender, sends the sequence of sound pulses into the sample $(\mathrm{H})$, figure 1 . In this system a single pulse is reflected back and forth in the sample creating a whole family of successive

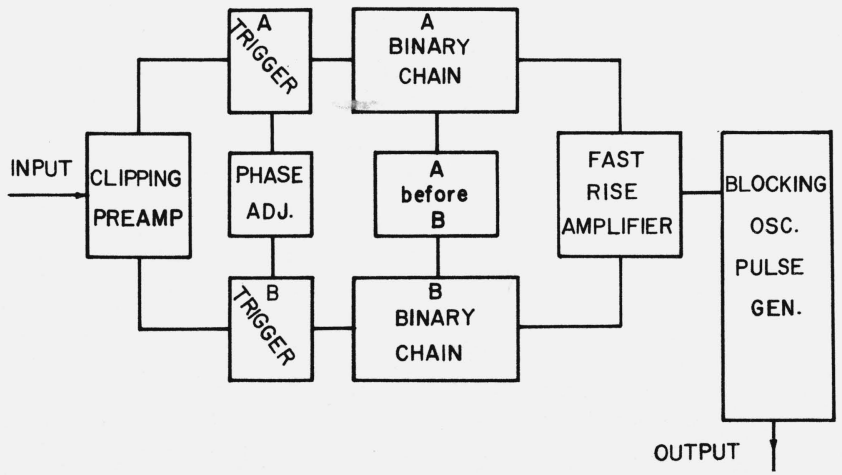

Figure 2. Two-shot pulse generator - a block diagram.

A before B switch sets the chain firing order, namely A before B or B before A. acoustic reflections: first round trip reflection, second round trip reflection, etc. In this way the two pulses as generated by the apparatus form two overlapping families of acoustical reflections. The superposition of all the acoustical reflections is received by the transducer. The electrical output of the transducer is amplified by a low noise amplifier ((D) of fig. 1) and then the coincidences are displayed on a delayed sweep $100 \mathrm{MHz}$ scope (E).

Coincidences are set between the two overlapped families of reflected pulses. With the control oscillator, the period $\tau$ is adjusted until the second reflection generated by the first pulse of the pair coincides with the first reflection generated by the second pulse. Specifically this coincidence is made by aligning the first half cycles of the two reflections. Usually the combination of the two first half cycles is maximized to indicate alinement. This represents a coincidence made at a time between $1 / 3$ to $1 / 2$ of the resonant period of the transducer after the receipt of the very first arrival (earlier coincidences can be obtained photographically). The definition of the coincidence setting is improved by a low frequency phase modulation of the control oscillator (fig. $1[\mathrm{~F}]$ modulation oscillator).

Because the two-shot apparatus affords a very low pulse rate, coincidences are especially easy to set even for materials of the highest $Q$. This condition is due primarily to the absence of driving energy on the transducers at the time of coincidence and to the lack of acoustical reverberation from the previous pulses afforded by the long dead time.

Very thin barium titanate transducers are used for both longitudinal and shear wave generation and reception (see fig. 3). A backing mixture of epoxy resin and tungsten graded powders is attached to the transducers. The high loss characteristics of this mixture helps keep the $Q$ of the transducer system below 10, which is desirable. This reduces long ringing and improves the initial pulse edge.

The transducers are coupled to the sample by an acoustically thick joint. Joints 10 to $25 \mu \mathrm{m}$ in thickness are constructed of materials having an acoustic impedance much lower than that of either the transducer or sample. This helps isolate the sample from the transducer and the reflections of sound energy in the sample tend to take place at the proper surface. These bonds have the advantage that they are simple and easy to construct. Compressional bonds are usually made with water or

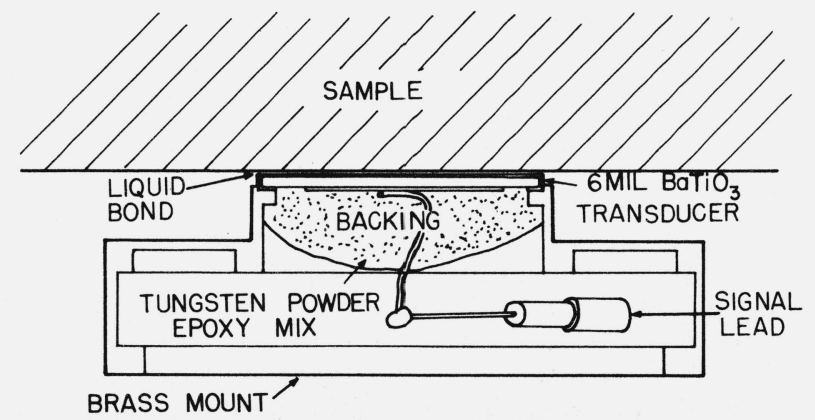

Figure 3. Typical transducer and bond construction. 
alcohol at room temperature and 4-methyl-1-pentene [10] or 2-methyl butane [11] at low temperature (77 to 196 $\mathrm{K})$. A good shear coupling agent at room temperature is Dow Resin 276-V9 [1] (poly-methyl styrene), and at low temperatures is 4-methyl-1-pentene.

\section{Technique Evaluation}

Errors and accuracy have been evaluated by a number of different experiments for which various accessible variables can be controlled. Two criteria are used for evaluation: sound speed and round-trip transit time. Round-trip transit time is used primarily for studying small time delays associated with bond and end effects. The sound speed is measured whenever the "Crucial Test" is applied.

\subsection{The Crucial Test (Sound Speed Uniformity)}

The accurate determination of sound speed is hindered greatly by the lack of a uniquely definitive experiment. In general, the toughest convenient test for accuracy that is readily available is to examine the change of sound speed as a function of sample length. In liquids this is relatively simple. The physical limitations of solid samples cause special problems. The following four are examples: (1) The length of a solid sample cannot be changed in a continuous fashion. (2) Material uniformity is always questionable. (3) Spurious reflections occur more readily in the smaller physical sizes commonly used in solid measurements. Finally (4) there are difficulties in bonding the transducer to the sample. The importance of the constancy of sound speed with length cannot be overemphasized. Although it is not a sufficient test, it must necessarily be applied whenever accuracy is of interest.

This test was applied for three different materials. The first sample used was a family of aluminum gage blocks (5 lengths $3 / 4$ in thru 4 in long $\times 2$ in diam). The blocks were cut consecutively from a polycrystalline mother bar. Under the best measurement conditions, the longitudinal speed of sound was found constant within $\pm 2 \times 10^{-4}$ parts (18 MHz resonant transducer) and the shear speed within $\pm 1 \times 10^{-3}$ parts $(8.5 \mathrm{MHz}$ resonant transducer) for the five lengths. These errors in sound speed for the aluminum sample could easily be explained by the lack of radial stress uniformity in the bar samples and by nonuniform sound scattering caused by the polycrystalline state.

A search for a better material was begun. Commercial grade fused silica was tried and found to be poor for the purpose. Local variations in the internal stress and density as seen in a polarimeter could be roughly correlated with variations in sound speed measured locally. Fused silica of more uniform optical density gave better uniformity in the sound speed. Finally a successful material was found in a borosilicate optical crown glass manufactured by the National Bureau of Standards under the name BSC 517/645. Some physical properties of this glass are given in the Smithsonian Physical Tables [12]. This material was found to have high optical and acoustical uniformity. At room temperature this glass has the bonus property of a near zero velocity temperature coefficient.

A test sample of this material was fabricated so as to have three different path lengths (a parallelepiped of 1 in $\times 1.5$ in $\times 2.0$ in). The question of acoustical uniformity is then isolated to local sound speed uniformity, and this has been determined to be below the threshold of measurement for this method $\left(< \pm 5 \times 10^{-5}\right.$ parts $)$. Results for compressional waves, using a transducer resonant at $18 \mathrm{MHz}$ on the $\mathrm{BSC} 517$ sample, showed a barely measurable increase in speed for shorter path lengths $\left[\mathrm{V}(1\right.$ in $)>\mathrm{V}(2$ in $)$ by $\sim 10^{-4}$ parts $]$. As will be discussed later, adjustments that reduced the bond error made the sound speed on this sample constant to the precision of the method. The same sample was used to test the shear mode (8.5 MHz resonant transducer) and the shear speed was found to be constant to within the precision of $\pm 5 \times 10^{-5}$ parts.

Using different path lengths, another material was measured. With a glass optical absorption cell (nominally 2 in by 3 in) the longitudinal $(18 \mathrm{MHz}$ resonant transducer) sound speed of pure water was measured along two path lengths (a third length was obtained from another cell). The transducers were fixed to the outside walls of the container in the same manner as they are for any solid. Thus solid bonding problems are duplicated here although these effects are smaller than normal because of the larger transit times involved. The temperature environment was well isolated and highly controlled. A platinum resistance thermometer was used to measure temperatures to a millidegree. Results on three path lengths $(6.3,8.9,14.0 \mathrm{~cm})$ indicate that the constancy of sound speed is better than the total precision $( \pm 5 \times$ $10^{-5}$ parts) and that the measured value agrees extremely well with the recent work of Carnvale et al. [13].

\subsection{Effect of Diameter of Transducer}

The effects of changing transducer diameter with constant frequency were investigated. Very thin paper masks $(25 \mu \mathrm{m})$ were used to restrict the radiating area of the transducer. Bonds were made by filling the hole in the mask with the bonding fluid. (It was determined that no sound could be transmitted through the wetted mask area.) Figure 4 shows how the mask hole size is related to the measured transit time through the sample. This experiment was performed on fused silica using three different resonant transducers. In general, the transit time increases (speed decreases) for smaller effective transducer areas. This behavior might be explained by the notions discussed in 4.1. The abrupt change of speed from the unmasked value to the largest masked value is due primarily to the thick bond effect which will be discussed later. When the same experiment was performed using shear waves, very little change in transit time was measured (less than $10^{-4}$ parts).

\subsection{Effect of Resonant Frequency of Transducer}

Early experiments on fused silica indicated that the measured sound speed tends to decrease with increasing resonant frequency of the transducer. A similar effect was found with the BSC 517/645 sample. Figure 5 shows the 


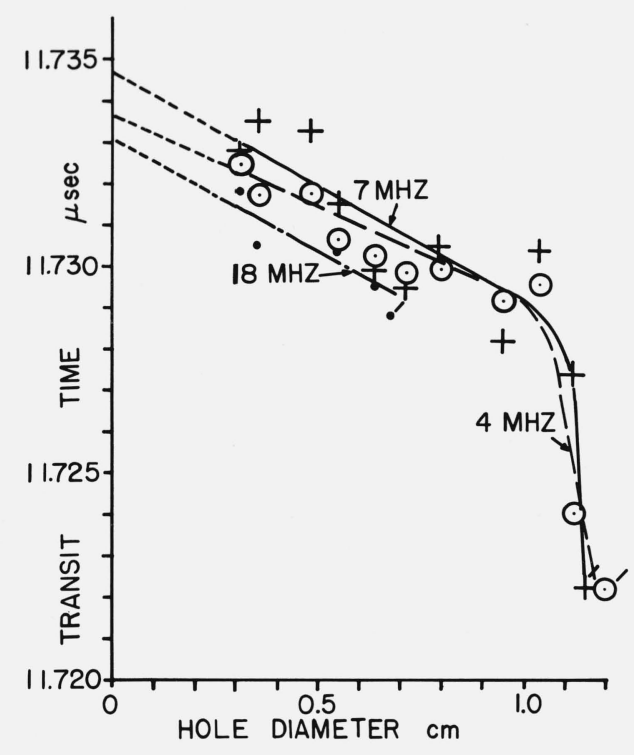

Figure 4. Effect of mask diameter on transit time.

3.5-cm long fused silica sample with each of three transducers of different frequency. Water forms the bond. Slashes mean "no mask."

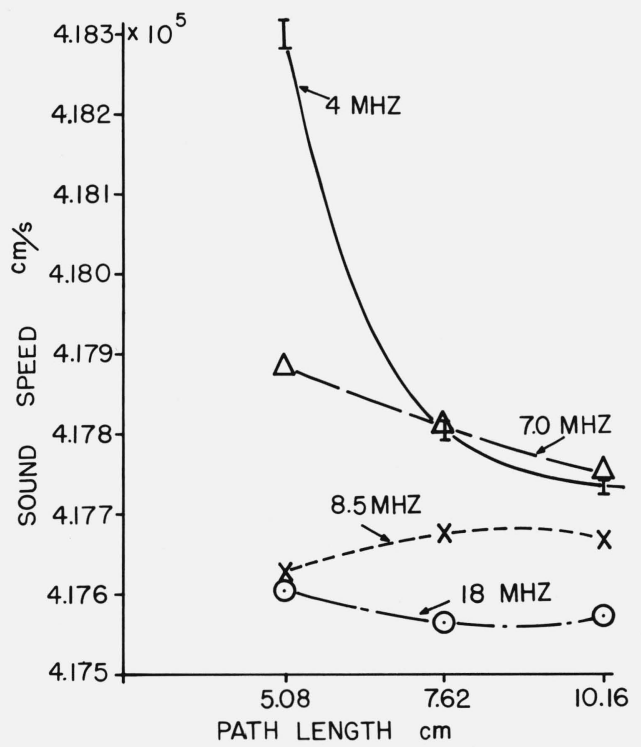

Figure 5. Sound speed (L-waves) in borosilicate crown glass BSC $517 / 645$ for three path lengths and four transducers of different frequency.

Tight water bond. Flags indicate precision.

constancy test of sound speed as performed with the three lengths of the borosilicate sample using four longitudinal transducers of different resonant frequency. The resonant frequency of each transducer is marked on the appropriate curve. Note that as the resonant frequency increases, the speed decreases and becomes more uniform with path length (maximum spread for all transducer frequencies and sample lengths is $2 \times 10^{-3}$ ). These measurements were made with a tight bond (water bonds of less than $10 \mu \mathrm{m}$ ).

\subsection{Earlier Coincidences}

As already mentioned in the introduction, it is felt that the closer to the first sound arrival that one sets the timing coincidences the better the resulting accuracy will be. One way to facilitate this is to use transducers of higher resonant frequency as was discussed in section 3.3. Another is to use earlier recognizable features of the transient pulse. Efforts were made to examine the effects on the transit time of the position of the coincidence setting in the received pulse. Transit times for longitudinal sound through a fused silica sample were measured for nine successive half cycles. The coincidences were set by maximizing each half cycle starting with the first and working back to the ninth half cycle in the pulse. These results are shown in figure 6 for two different resonant frequency transducers. The various half cycles at which coincidences were made are appropriately marked and the position of each related to the beginning of the pulse is measured on the abscissa in $\mu \mathrm{s}$. This test was also performed using shear waves $(8.5 \mathrm{MHz}$ resonant transducer) with very similar results.

An arbitrary scheme of obtaining information about coincidences earlier than the first half cycle can be carried out photographically. Photos of the front edge of the first half cycle were obtained from a $100 \mathrm{MHz}$ fast sweep scope. Pictures were taken for both the first and second reflections separately. Amplitude measurements were taken from these as a function of time back to the beginning of the signal from the first half cycle maximum. A

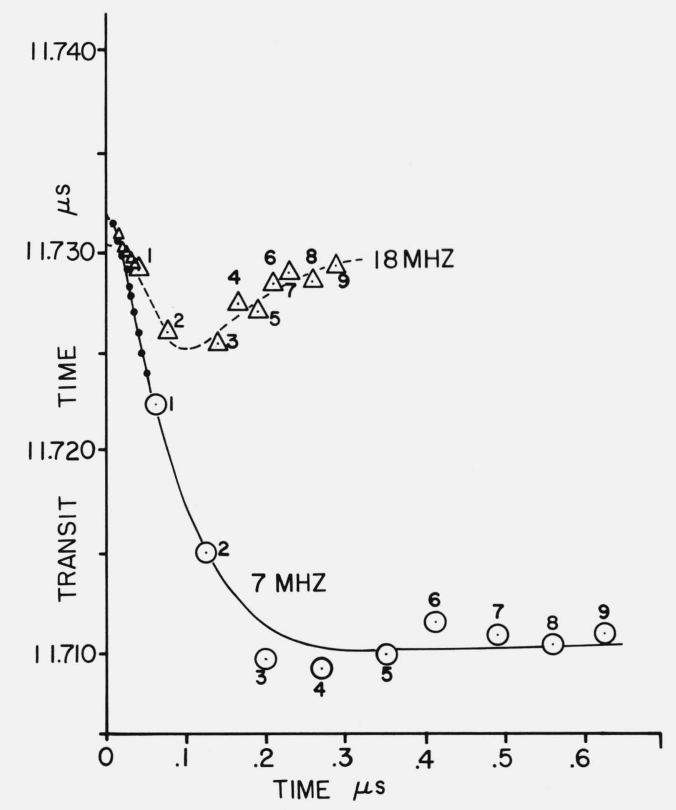

Figure 6. Transit time (L-waves) versus time from the beginning of the signal at which coincidence was made.

Two transducers (18 and $7 \mathrm{MHz}$ resonance frequency) on $3.5-\mathrm{cm}$ long fused silica sample. Tight water bond. 


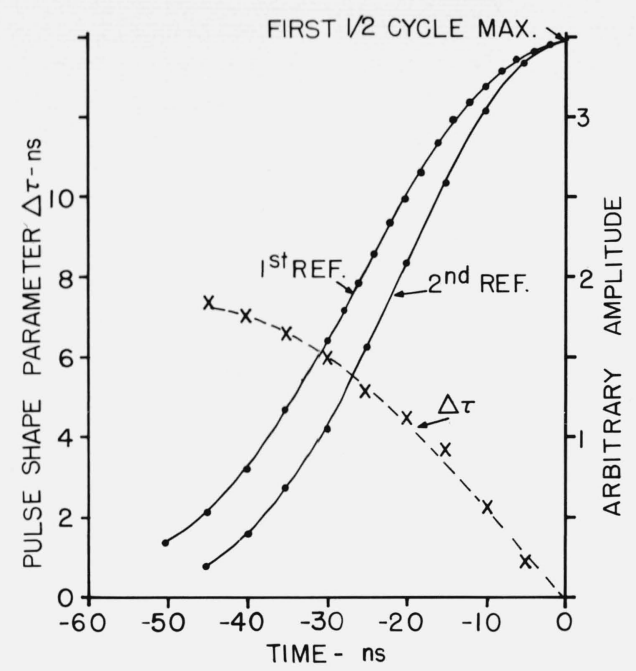

Figure 7. First quarter cycle of first and second reflections normalized at the first $1 / 2$ cycle maximum.

$\Delta \tau$ is excess delay for points of equal amplitude (arbitrary time zero at first $1 / 2$ cycle max.) L-waves in $2.5-\mathrm{cm}$ long borosilicate crown glass. Transducer, $7 \mathrm{MHz}$. Tight water bond.

normalized amplitude graph demonstrates any shape change between the front edges of the first half cycle for the first and second reflections.

Figure 7 is one such normalized comparison. The time reference origin is fixed at the first maximum of each and negative time proceeds forward into the pulse in the normal way. Note that in this case the second reflection rises faster than the first (indicative of some geometric interference effects). The time difference between equal amplitude points of the two pulses is plotted as $\Delta \tau$, the pulse shape parameter. This is associated with pulse shape change and has been used to correct the first half cycle coincidence data to give better values of sound speed. As one goes forward into the pulse the precision of $\Delta \tau$ gets steadily worse making values of $\Delta \tau$ earlier than $30 \mathrm{~ns}$ from the first half cycle peak undependable.

Using $\Delta \tau$ to correct the transit times, corrections can be found for the sound speed. These corrected transit times are shown by the small $\Delta$ data and the $\bullet$ data for the two different resonant frequency transducers on figure 6 . Note that these data tend to converge to the same value at the pulse beginning. Further confirmation for this arbitrary correction is obtained from the favorable results of sound speed uniformity tests shown in figure 8. Figure 8 is figure 4 corrected for the early pulse shape variations using $\Delta \tau$ for the three different path lengths and the four resonant transducers on the BSC 517/645 sample. Note that not only is the sound speed more nearly constant for the three lengths, but the data for the different resonant frequency transducers tends to cluster around the $18 \mathrm{MHz}$ transducer value (maximum spread for all lengths and frequencies is $\left.2.5 \times 10^{-4}\right)$. In the case of shear waves, the difference in shape of the first two reflections is undetectable and there is no reason for such a correction.

The following general observations can be made about the behavior of $\Delta \tau$, pulse shape, and sound speed.

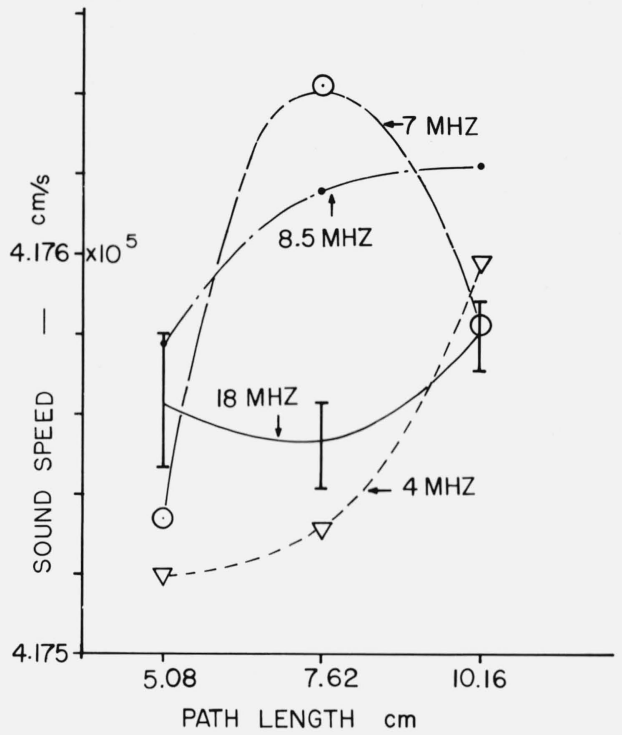

Figure 8. Speed of sound (L-waves) in borosilicate crown glass BSC 517/645 for three path lengths and four transducers of different frequency.

The data are those of figure 4 corrected according to figure 7 . Flags indicate precision.

(1) The higher the resonant frequency of the transducer the smaller the pulse shape factor. In fact for the $18 \mathrm{MHz}$ resonant transducer $\Delta \tau$ is essentially zero.

(2) The smaller $\Delta \tau$ is (or the more nearly alike the first and second reflections are) the more uniform is the sound speed for the three path lengths.

(3) By applying the corrections for the shape factor to the sound speed, the constancy is improved and the values of sound speed, as corrected, bunch closer to the highest frequency value.

\subsection{Transducer-Sample Bonds}

The effects of a thick bond on the transit time for longitudinal waves were studied by using a variable water bond. Apparatus that allowed continuous fine adjustment of the bond thickness while maintaining parallelism between the transducer and sample was used. Changes in the transit time were measured as a function of bond thickness for a number of different materials, a number of path lengths and different frequency transducers. Figure 9 is typical. It shows the increase in the measured transit time with bond thickness for three transducers of different resonant frequencies. The three path BSC 517 sample was used. Measurements along these three path lengths give essentially the same increase in transit time, indicating that the bond errors are independent of sample length. Furthermore, using transducers of higher resonant frequency reduces the magnitude of the bond error. Similar work using the $8.5 \mathrm{MHz}$ shear resonant transducer with a viscous resin bonding material (Dow 276-V9 Resin) was done. A similar but much smaller increase in transit time was obtained (increase was $\sim 1 \times 10^{-4}$ parts). 


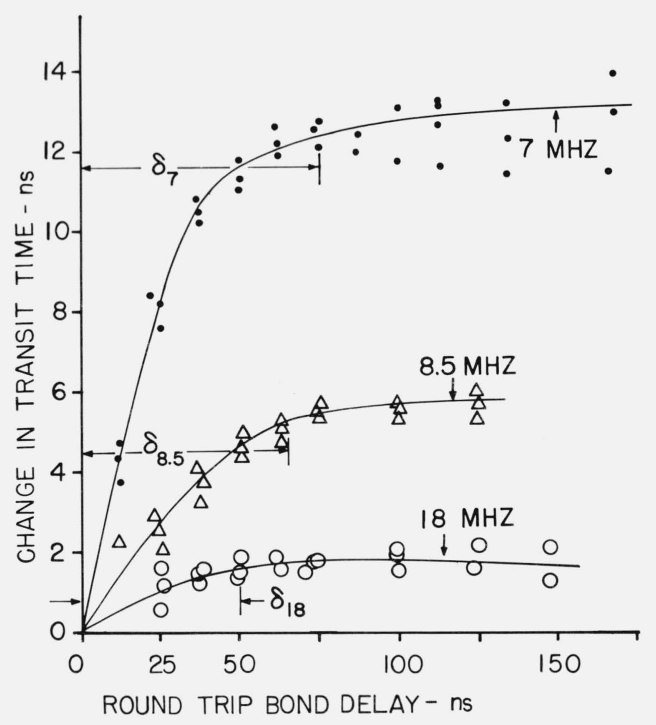

Figure 9. Change in transit time versus water bond thickness. L-waves in borosilicate crown glass BSC 517/645. Three transducers of different frequency. Data for three lengths on each curve. $\delta$ is the half period of the transducer frequency.

Generally as the thickness is increased to that of the $\delta$ bond (thickness corresponding to the time between the first arrival and the first maximum) both the constancy of the sound speed and pulse shape factor are improved. In fact when the sound speed is measured on the three length BSC 517 sample using $\delta$ bonds, in a similar manner to figure 4 , one finds that all the transducers of different resonant frequencies give more constant values of sound speed and the values obtained cluster much closer to each other than for a thin bond (maximum spread for all frequencies and lengths is $\left.1.5 \times 10^{-4}\right)$. At the highest transducer frequency of $18 \mathrm{MHz}$, measurement of sound speed with a $\delta$ bond gives values constant within $\pm 5 \times$ $10^{-5}$ parts which is lower by $10^{-4}$ parts than the data of figure 4 , taken with thin bond.

\subsection{Damping Backing to Transducer}

Interference in the leading edge of the received pulses which occurs because of reflections off the back of the transducers was investigated. A number of different backing mixtures were tried in an effort to reduce transducer ringing. It was found that lowering the $Q$ of the transducer by use of a backing with higher damping improved its performance. Both the pulse shape and the constancy of sound speed were improved by a tungsten-epoxy backing which lowered the $Q$ to less than 10 . At $18 \mathrm{MHz}$ pulse shapes were made nearly identical by the use of these improved backings.

\section{Discussion}

\subsection{Expected Interference Errors}

It is difficult to estimate theoretically the magnitude and the sign of errors in sound speed unless sufficient knowledge about the particular measurement is available. In these experiments, coincidences are made between corresponding points on the front edge of the first and second reflections. Depending on the kind of distortion acquired between these reflections, error of either sign may be expected in the transit time.

Figure 10A represents the condition observed experimentally by this method at the lower frequencies. This shows the leading edges of the first and second reflections as they are related to the first time of arrival. The first reflection rises more slowly to the peak than does the second. The contrary situation has never been observed in all the experiments performed. Let the measured transit time, $X$, be given by

$$
X=\tau+\Delta t_{2}-\Delta t_{1},
$$

where $\tau$ is the shortest time of flight, associated with first arrival. Because $\Delta t_{2}$, the time between the first arrival and the first maximum of the second reflection, is smaller than $\Delta t_{1}$, the corresponding time for the first reflection, the measured transit time will be too small when coincidences of the first half cycle are used. Thus, improving the similarity between pulses of the first and second reflections should cause the transit time to increase. This is what is observed experimentally. In fact, it occurs in conjunction with the improvement of sound speed constancy. The following simplification may help to explain the observed differences in the first and second pulse reflections.

Assume that for the leading edge of the signal the sender acts more like a point source than like a plane wave generator. Figure $10 \mathrm{~B}$ shows the longest and the shortest rays that can connect a point sender and a finite planar receiver for the first two reflections. The difference between the longest received and most direct rays is

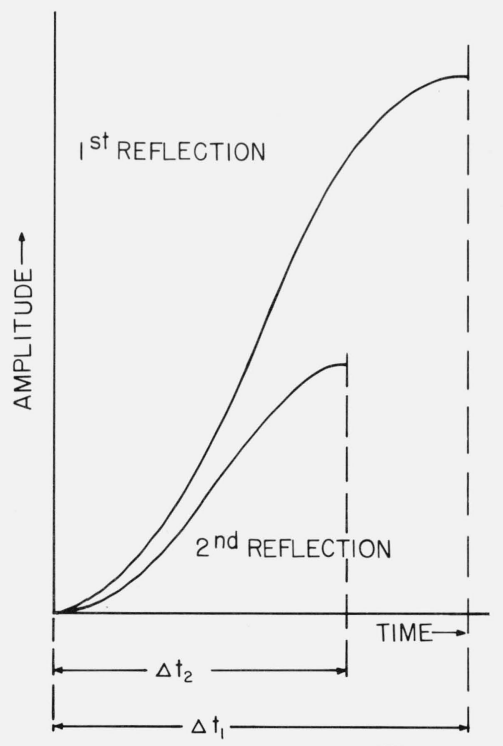

Figure 10-A. Front edge of the first and second reflections. Time versus amplitude. First arrival is at the origin. 


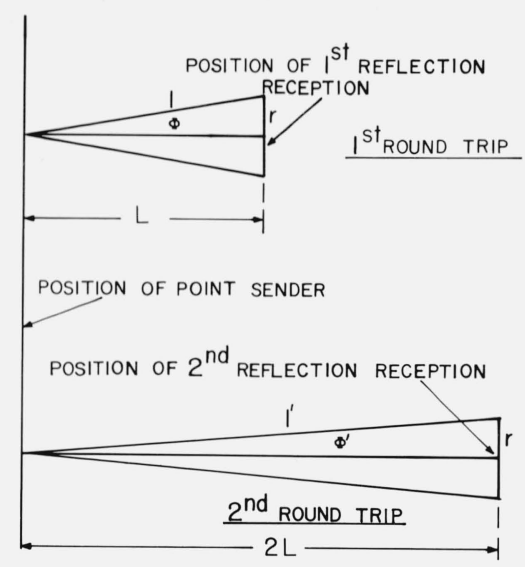

Figure 10-B. Extreme rays connecting a point sender to a finite receiver for two consecutive reflections.

greater for the first reflection than for the second. Thus one might expect the delay between the first arrival and the initial maximum to be greater for the first reflection than for the second. Although this model fits the qualitative behavior, it oversimplifies a much more complicated geometric interference problem. It is for this and similar reasons that the empirical test has become the guideline for this experiment.

\subsection{Bond Errors}

In this method one of the biggest sources of error concerns multiple reflections between the two faces of the bond. When sound is sent through a bond of finite thickness some of the energy is transmitted and some internally reflected. In such a manner a whole family of pulses separated by some multiple of twice the transit time of the bond will be transmitted into the sample. The superposition of these reflections changes the shape of the observed pulse. The resultant shape depends on how the intrabond reflections add to the straight-through pulse. The leading edge of the pulse may be sharpened, lengthened, or left essentially unchanged. A similar process will occur at the encounter of the sound with the bond after each round trip.

Not all of the encounters are of the same kind. The most important of these is the one after the sound has completed the first round trip. Part of the sound is transmitted through the bond and the rest is reflected back into the sample. With a thin bond, there are multiple reflections at the faces of the bond which modify both the reflected and transmitted waves. Experiment indicates that the signal received by the transducer after one round trip is lengthened by more than the geometrical effect discussed above. On the other hand, the signal received after two or more round trips is modified less by the bond.

To avoid the influences of these intrabond reflections, a thick bond is used. This " $\delta$ bond" delays the unwanted reflections so coincidences formed from the direct pulses will be unhindered by later reflections. Experimentally, when the $\delta$ bond is used, one finds that the constancy of sound speed is improved, that the shape differences between first and second reflections become less and that the transit times are longer (measured speeds are lower) than for a thin bond.

\section{Summary}

The transient pulse technique of sound speed measurement has been shown to be highly accurate when used on nondispersive materials. Accuracies of the order of the precision, which is $\pm 5 \times 10^{-5}$, have been realized. The constancy of sound speed was used as the measure of accuracy. The accuracy of this technique is affected by a number of variables. The observed sound speeds are more nearly constant when using: (a) higher resonant frequency transducers, (b) earlier data in the pulse to set coincidences, (c) thick buffer bonds of $\delta$ thickness, which remove interference caused by the intrabond reflections, and (d) transducers of smaller diameter-tothickness ratio. The obvious has been confirmed in that, as the sound speed constancy is improved, the leading edges of the first two reflections become more identical. Local acoustical uniformity of the test samples is very important and the NBS optical glass BSC 517 fills these requirements quite well. The choice of a multipath sample as opposed to many samples of different length removes much of the question of sample uniformity.

The author is deeply indebted to Martin Greenspan and Frank Breckenridge for discussions about the acoustical aspects, to Carl Tschiegg for help with certain electronic features, and to J. Wilson and C. Smith for typing. This work was supported in part by the U.S. Office of Naval Research.

\section{References}

[1] McSkimin, H. J., Measurements of elastic constants at low temperatures by means of ultrasonic waves, J. Appl. Phys. 24, 988-997 (1953).

[2] McSkimin, H. J., Measurement of ultrasonic wave velocities and elastic moduli for small solid specimens at high temperature, J. Acoust. Soc. Am. 31, 287-295 (1959) .

[3] McSkimin, H. J., Empirical study of the effect of diffraction on velocity of propagation of high frequency ultrasonic waves, J. Acoust. Soc. Am. 32, 1401-1404 (1960).

[4] McSkimin, H. J., Measurement of the $25^{\circ} \mathrm{C}$ zero-field elastic moduli of quartz by high frequency plane-wave propagation. J. Acoust. Soc. Am. 34, 1271-1274 (1962).

[5] Papadakis, E. P., Ultrasonic phase velocity by the pulseecho-overlap method incorporating diffraction phase corrections, J. Acoust. Soc. Am. 42, 1045-1051 (1967)

[6] Greenspan, M., and Tschiegg, C. E., Sing around ultrasonic velocimeter for liquids, Rev. Sci. Instr. 28, 897-901 (1957).

[7] Greenspan, M., and Tschiegg, C. E., Speed of sound in water by a direct method, J. Res. Nat. Bur. Stand. (U.S.), 59, 249-254 (1957).

[8] Proctor, T. M., Low-temperature speed of sound in singlecrystal ice, J. Acoust. Soc. Am. 39, 972-977 (1966).

[9] Tschiegg, C. E., and Greenspan, M., An accurate time modulated pulse circuit, Rev. Sci. Instr. 24, 183 (1953). 
[10] Bateman, T. B., Some materials for ultrasonic transducer bonding at cryogenic temperatures, J. Acoust. Soc. Am. 41, 1011-1014 (1966).

[11] Stern, R., Natale, G. G., and Rudnick, I., Isopentane bond for low temperature ultrasonics, Rev. Sci. Instr. 35, 10771078 (1964).
[12] Forsythe, W. E., Smithsonian Physical Tables, The Lord Baltimore Press, 1954.

[13] Carnvale, A., Bower, P., Basileo, M., and Sprenke, J., Absolute sound-velocity measurement in distilled water, J. Acoust. Soc. Am. 44, 1098-1102 (1968) .

(Paper 75Cl-312) 